General Mathematics Vol. 28, No. 2 (2020), 3-17

DOI: $10.2478 / \mathrm{gm}-2020-0011$

S sciendo

\title{
Some Bullen-type inequalities for conformable fractional integrals ${ }^{1}$
}

\author{
Musa Çakmak
}

\begin{abstract}
In this study, the author has established a new lemma and Bullen-type inequalities for conformable fractional integrals. Also, it is given some applications involving Bullen type integral inequalities for differentiable functions to show the results.
\end{abstract}

2010 Mathematics Subject Classification: 26D07, 26D15.

Key words and phrases: Bullen type inequality, Conformable fractional integrals, Hölder's inequality, Power-mean inequality.

\section{Introduction}

The Hermite-Hadamard inequality: Let $\Lambda: J \subseteq \mathbb{R} \rightarrow \mathbb{R}$ be a convex function and $u_{1}, u_{2} \in J$ with $u_{1}<u_{2}$.

$$
\Lambda\left(\frac{u_{1}+u_{2}}{2}\right) \leq \frac{1}{u_{2}-u_{1}} \int_{u_{1}}^{u_{2}} \Lambda(\varkappa) d \varkappa \leq \frac{\Lambda\left(u_{1}\right)+\Lambda\left(u_{2}\right)}{2}
$$

If $\Lambda$ is concave, this double inequality hold in the inverse way. See $[7,11,17,19]$ for details.

The Bullen inequality:

$$
\frac{1}{u_{2}-u_{1}} \int_{u_{1}}^{u_{2}} \Lambda(\varkappa) d \varkappa \leq \frac{1}{2}\left[\frac{\Lambda\left(u_{1}\right)+\Lambda\left(u_{2}\right)}{2}+\Lambda\left(\frac{u_{1}+u_{2}}{2}\right)\right],
$$

provided that $\Lambda:\left[u_{1}, u_{2}\right] \rightarrow \mathbb{R}$ is a convex function on $\left[u_{1}, u_{2}\right]$ (see for example $[12,13,20,27])$ for details. In [14], s-convexity is defined by Hudzik and Maligranda

\footnotetext{
${ }^{1}$ Received 17 June, 2019

Accepted for publication (in revised form) 23 April, 2020
} 
as follows: a function $\Lambda: \mathbb{R}_{+} \rightarrow \mathbb{R}$, where $\mathbb{R}_{+}=[0, \infty)$, is said to be $s$-convex in the second sense if

$$
\Lambda(\alpha x+(1-\alpha) y) \leq \alpha^{s} \Lambda(x)+(1-\alpha)^{s} \Lambda(y)
$$

for all $x, y \in[0, \infty), \alpha \in[0,1]$ and for some fixed $s \in(0,1]$.

Lemma 1 [10] Let $\Lambda: J \subseteq \mathbb{R} \rightarrow \mathbb{R}$ be differentiable function on $J^{\circ}, u_{1}, u_{2} \in J$, with $u_{1}<u_{2}$. If $\Lambda^{\prime} \in L\left[u_{1}, u_{2}\right]$ and $t \in[0,1]$ then

$$
\begin{aligned}
& \frac{u_{2}-u_{1}}{8}\left[\int_{0}^{1}(1-2 t) \Lambda^{\prime}\left(\frac{1+t}{2} u_{1}+\frac{1-t}{2} u_{2}\right) d t\right. \\
& +\int_{0}^{1}(2 t-1) \Lambda^{\prime}\left(\frac{1+t}{2} u_{2}+\frac{1-t}{2} u_{1}\right) d t \\
= & \frac{1}{2}\left[\frac{\Lambda\left(u_{1}\right)+\Lambda\left(u_{2}\right)}{2}+\Lambda\left(\frac{u_{1}+u_{2}}{2}\right)\right]-\frac{1}{u_{2}-u_{1}} \int_{u_{1}}^{u_{2}} \Lambda(\varkappa) d \varkappa .
\end{aligned}
$$

Theorem 1 [10] Let $\Lambda: J \subseteq \mathbb{R} \rightarrow \mathbb{R}$ be differentiable function on $J^{\circ}, u_{1}, u_{2} \in J$, and $\Lambda^{\prime} \in L\left[u_{1}, u_{2}\right]$. If $\left|\Lambda^{\prime}(\varkappa)\right|^{q}$ is s-convex function in the second sense on $\left[u_{1}, u_{2}\right]$ for some fixed $s \in(0,1], p, q>1,1 / p+1 / q=1$, then

$$
\begin{gathered}
\quad\left|\frac{1}{2}\left[\frac{\Lambda\left(u_{1}\right)+\Lambda\left(u_{2}\right)}{2}+\Lambda\left(\frac{u_{1}+u_{2}}{2}\right)\right]-\frac{1}{u_{2}-u_{1}} \int_{u_{1}}^{u_{2}} \Lambda(\varkappa) d \varkappa\right| \\
\leq \frac{u_{2}-u_{1}}{8}\left(\frac{1}{p+1}\right)^{\frac{1}{p}}\left[\left(\frac{2-\left(\frac{1}{2}\right)^{s}}{s+1}\left|\Lambda^{\prime}\left(u_{1}\right)\right|^{q}+\frac{\left(\frac{1}{2}\right)^{s}}{s+1}\left|\Lambda^{\prime}\left(u_{2}\right)\right|^{q}\right)^{\frac{1}{q}}\right. \\
\left.+\left(\frac{\left(\frac{1}{2}\right)^{s}}{s+1}\left|\Lambda^{\prime}\left(u_{1}\right)\right|^{q}+\frac{2-\left(\frac{1}{2}\right)^{s}}{s+1}\left|\Lambda^{\prime}\left(u_{2}\right)\right|^{q}\right)^{\frac{1}{q}}\right] .
\end{gathered}
$$

If taking $s=1$ in Theorem 1 , the following corollary derived.

Corollary 1 [10] Let $\Lambda: J \subseteq \mathbb{R} \rightarrow \mathbb{R}$ be differentiable function on $J^{\circ}, u_{1}, u_{2} \in J$ and $\Lambda^{\prime} \in L\left[u_{1}, u_{2}\right]$. If $\left|\Lambda^{\prime}(\varkappa)\right|^{q}$ is convex function on $\left[u_{1}, u_{2}\right]$ and $1 / p+1 / q=1$, then

$$
\begin{aligned}
& \left|\frac{1}{2}\left[\frac{\Lambda\left(u_{1}\right)+\Lambda\left(u_{2}\right)}{2}+\Lambda\left(\frac{u_{1}+u_{2}}{2}\right)\right]-\frac{1}{u_{2}-u_{1}} \int_{u_{1}}^{u_{2}} \Lambda(\varkappa) d \varkappa\right| \\
\leq & \frac{u_{2}-u_{1}}{8(p+1)^{1 / p} 4^{1 / q}} \times \\
& {\left[\left(3\left|\Lambda^{\prime}\left(u_{1}\right)\right|^{q}+\left|\Lambda^{\prime}\left(u_{2}\right)\right|^{q}\right)^{\frac{1}{q}}+\left(\left|\Lambda^{\prime}\left(u_{1}\right)\right|^{q}+3\left|\Lambda^{\prime}\left(u_{2}\right)\right|^{q}\right)^{\frac{1}{q}}\right] . }
\end{aligned}
$$


Theorem 2 [10] Let $\Lambda: J \subseteq \mathbb{R} \rightarrow \mathbb{R}$ be differentiable function on $J^{\circ}, u_{1}, u_{2} \in J$, and $\Lambda^{\prime} \in L\left[u_{1}, u_{2}\right]$. If $\left|\Lambda^{\prime}(\varkappa)\right|^{q}$ is s-convex function in the second sense on $\left[u_{1}, u_{2}\right]$ for $q \geq 1$, then

$$
\left|\frac{1}{2}\left[\frac{\Lambda\left(u_{1}\right)+\Lambda\left(u_{2}\right)}{2}+\Lambda\left(\frac{u_{1}+u_{2}}{2}\right)\right]-\frac{1}{u_{2}-u_{1}} \int_{u_{1}}^{u_{2}} \Lambda(\varkappa) d \varkappa\right|
$$

$\leq \frac{u_{2}-u_{1}}{8}\left(\frac{1}{2}\right)^{1-1 / q} \times$

$$
\begin{aligned}
& \left\{\left[\frac{\left(9\left(\frac{3}{2}\right)^{s}-4 \times 2^{s}+2 \times 2^{s} s-s-4\right)}{2^{s}(s+1)(s+2)}\left|\Lambda^{\prime}\left(u_{1}\right)\right|^{q}+\frac{s+\frac{1}{2^{s}}}{2^{s}(s+1)(s+2)}\left|\Lambda^{\prime}\left(u_{2}\right)\right|^{q}\right]^{1 / q}\right. \\
& \left.+\left[\frac{\left(9\left(\frac{3}{2}\right)^{s}-4 \times 2^{s}+2 \times 2^{s} s-s-4\right)}{2^{s}(s+1)(s+2)}\left|\Lambda^{\prime}\left(u_{2}\right)\right|^{q}+\frac{s+\frac{1}{2^{s}}}{2^{s}(s+1)(s+2)}\left|\Lambda^{\prime}\left(u_{1}\right)\right|^{q}\right]^{1 / q}\right\}
\end{aligned}
$$

where

$$
\begin{aligned}
\frac{1}{2^{s}} \int_{0}^{1}|1-2 t|(1+t)^{s} d t & =\frac{\left(9\left(\frac{3}{2}\right)^{s}-4 \times 2^{s}+2 \times 2^{s} s-s-4\right)}{2^{s}(s+1)(s+2)} \\
\frac{1}{2^{s}} \int_{0}^{1}|1-2 t|(1-t)^{s} d t & =\frac{s+\frac{1}{2^{s}}}{2^{s}(s+1)(s+2)}
\end{aligned}
$$

and

$$
\int_{0}^{1}|1-2 t| d t=\frac{1}{2}
$$

If taking $s=1$ in Theorem 2, we derive the following corollary.

Corollary 2 [10]Let $\Lambda: J \subseteq \mathbb{R} \rightarrow \mathbb{R}$ be differentiable function on $J^{\circ}, u_{1}, u_{2} \in J$ and $\Lambda^{\prime} \in L\left[u_{1}, u_{2}\right]$. If $\left|\Lambda^{\prime}(\varkappa)\right|^{q}$ is convex function on $\left[u_{1}, u_{2}\right]$ and $q \geq 1$, then

$$
\begin{aligned}
& \left|\frac{1}{2}\left[\frac{\Lambda\left(u_{1}\right)+\Lambda\left(u_{2}\right)}{2}+\Lambda\left(\frac{u_{1}+u_{2}}{2}\right)\right]-\frac{1}{u_{2}-u_{1}} \int_{u_{1}}^{u_{2}} \Lambda(\varkappa) d \varkappa\right| \\
\leq & \frac{u_{2}-u_{1}}{8}\left(\frac{1}{2}\right)^{1-1 / q} \times \\
& \left\{\left[\frac{3}{8}\left|\Lambda^{\prime}\left(u_{1}\right)\right|^{q}+\frac{1}{8}\left|\Lambda^{\prime}\left(u_{2}\right)\right|^{q} d t\right]^{1 / q}+\left[\frac{3}{8}\left|\Lambda^{\prime}\left(u_{2}\right)\right|^{q}+\frac{1}{8}\left|\Lambda^{\prime}\left(u_{1}\right)\right|^{q} d t\right]^{1 / q}\right\} .
\end{aligned}
$$

\section{Definition and Properties of Conformable Fractional Derivative and Integral}

The following definitions and theorems with respect to conformable fractional derivative and integral were referred in [1]-[6] and [8, 9, 15, 16, 21, 22, 23, 24, 25]. 
Definition 1 (Conformable fractional derivative) Given a function $\Lambda:[0, \infty) \rightarrow \mathbb{R}$. Then the "conformable fractional derivative" of $\Lambda$ of order $\alpha$ is defined by

$$
D_{\alpha}(\Lambda)(t)=\lim _{\varepsilon \rightarrow 0} \frac{\Lambda\left(t+\varepsilon t^{1-\alpha}\right)-\Lambda(t)}{\varepsilon}
$$

for all $t>0, \alpha \in(0,1]$. If $\Lambda$ is $\alpha$-differentiable in some $\left(0, u_{1}\right), \alpha>0, \lim _{t \rightarrow 0^{+}} \Lambda^{(\alpha)}(t)$ exist, then define

$$
\Lambda^{(\alpha)}(0)=\lim _{t \rightarrow 0^{+}} \Lambda^{(\alpha)}(t) .
$$

We can write $\Lambda^{(\alpha)}(t)$ for $D_{\alpha}(\Lambda)(t)$ to denote the conformable fractional derivatives of $\Lambda$ of order $\alpha$. In addition, if the conformable fractional derivative of $\Lambda$ of order $\alpha$ exists, then we simply say $\Lambda$ is $\alpha$-differentiable.

Theorem 3 Let $\alpha \in(0,1]$ and $\Lambda, g$ be $\alpha$-differentiable at a point $t>0$. Then,

1) $D_{\alpha}\left(u_{1} \Lambda+u_{2} g\right)=u_{1} D_{\alpha}(\Lambda)+u_{2} D_{\alpha}(g)$, for all $u_{1}, u_{2} \in \mathbb{R}$,

2) $D_{\alpha}(\lambda)=0$, for all constant functions $\Lambda(t)=\lambda$,

3) $D_{\alpha}(\Lambda g)=\Lambda D_{\alpha}(g)+g D_{\alpha}(\Lambda)$,

4) $D_{\alpha}\left(\frac{\Lambda}{g}\right)=\frac{D_{\alpha}(\Lambda) g+D_{\alpha}(g) \Lambda}{g^{2}}$

5) If $\Lambda$ is differentiable, then

$$
D_{\alpha}(\Lambda)(t)=t^{1-\alpha} \frac{d \Lambda}{d t}(t)
$$

Also,
a) $D_{\alpha}(1)=0$
b) $D_{\alpha}\left(e^{u_{1} t}\right)=u_{1} t^{1-\alpha} e^{u_{1} t}, u_{1} \in \mathbb{R}$
c) $D_{\alpha}\left(\sin \left(u_{1} t\right)\right)=u_{1} t^{1-\alpha} \cos \left(u_{1} t\right), u_{1} \in \mathbb{R}$
d) $D_{\alpha}\left(\cos \left(u_{1} t\right)\right)=-u_{1} t^{1-\alpha} \sin \left(u_{1} t\right), u_{1} \in \mathbb{R}$
e) $D_{\alpha}\left(\frac{1}{\alpha} t^{\alpha}\right)=1$
f) $D_{\alpha}\left(\sin \left(\frac{t^{\alpha}}{\alpha}\right)\right)=\cos \left(\frac{t^{\alpha}}{\alpha}\right)$
g) $D_{\alpha}\left(\cos \left(\frac{t^{\alpha}}{\alpha}\right)\right)=-\sin \left(\frac{t^{\alpha}}{\alpha}\right)$
h) $D_{\alpha}\left(e^{\frac{t^{\alpha}}{\alpha}}\right)=e^{\frac{t^{\alpha}}{\alpha}}$.

Theorem 4 (Mean value theorem for conformable fractional differentiable functions) Let $\alpha \in(0,1]$ and $\Lambda:[0, \infty) \rightarrow \mathbb{R}$ be a continuous on $\left[u_{1}, u_{2}\right]$ and an $\alpha$-fractional differentiable mapping on $\left(u_{1}, u_{2}\right)$ with $0 \leq u_{1}<u_{2}$. Then, there exist $c \in\left(u_{1}, u_{2}\right)$, such that

$$
D_{\alpha}(\Lambda)(c)=\frac{\Lambda\left(u_{2}\right)-\Lambda\left(u_{1}\right)}{\frac{u_{2}^{\alpha}}{\alpha}-\frac{u_{1}^{\alpha}}{\alpha}} .
$$

Definition 2 (Conformable fractional integral) Let $\alpha \in(0,1]$ and $0 \leq u_{1}<u_{2}$. A fucntion $\Lambda:[0, \infty) \rightarrow \mathbb{R}$ is $\alpha$-fractional integrable on $\left[u_{1}, u_{2}\right]$ if the integral

$$
\int_{u_{1}}^{u_{2}} \Lambda(x) d_{\alpha} x:=\int_{u_{1}}^{u_{2}} \Lambda(x) x^{\alpha-1} d x,
$$


exists and is finite. All $\alpha$-fractional integrable on $\left[u_{1}, u_{2}\right]$ is indicated by $L_{\alpha}^{1}\left(\left[u_{1}, u_{2}\right]\right)$.

\section{Remark 1}

$$
I_{\alpha}^{u_{1}}(\Lambda)(t)=I_{1}^{u_{1}}\left(t^{\alpha-1} \Lambda\right)=\int_{u_{1}}^{t} \frac{\Lambda(x)}{x^{1-\alpha}} d x
$$

where the integral is the usual Riemann improper integral and $\alpha \in(0,1]$.

Theorem 5 Let $\Lambda:\left(u_{1}, u_{2}\right) \rightarrow \mathbb{R}$ be differentiable and $\alpha \in(0,1]$. Then, for all $t>u_{1}$ we have

$$
I_{\alpha}^{u_{1}} D_{\alpha}^{u_{1}}(\Lambda)(t)=\Lambda(t)-\Lambda\left(u_{1}\right)
$$

Theorem 6 (Integration by parts). Let $\Lambda, g:\left[u_{1}, u_{2}\right] \rightarrow \mathbb{R}$ be two functions such that $\Lambda g$ is differentiable. Then,

$$
\begin{aligned}
& \int_{u_{1}}^{u_{2}} \Lambda(x) D_{\alpha}^{u_{1}}(g)(x) d_{\alpha} x \\
= & \left.\Lambda g\right|_{u_{1}} ^{u_{2}}-\int_{u_{1}}^{u_{2}} g(x) D_{\alpha}^{u_{1}}(\Lambda)(x) d_{\alpha} x .
\end{aligned}
$$

Theorem 7 Assume that $\Lambda:\left[u_{1}, \infty\right) \rightarrow \mathbb{R}$ such that $\Lambda^{(n)}(t)$ is continuous and $\alpha \in(n, n+1]$. Then, for all $t>u_{1}$ we have

$$
D_{\alpha}^{u_{1}}(\Lambda)(t) I_{\alpha}^{u_{1}}=\Lambda(t)
$$

Theorem 8 Let $\alpha \in(0,1]$ and $\Lambda:\left[u_{1}, u_{2}\right] \rightarrow \mathbb{R}$ be a continuous on $\left[u_{1}, u_{2}\right]$ with $0 \leq u_{1}<u_{2}$. Then,

$$
\left|I_{\alpha}^{u_{1}}(\Lambda)(x)\right| \leq I_{\alpha}^{u_{1}}|\Lambda|(x) .
$$

For more details and properties concerning the conformable integral operators, we refer, for example, to the works [1]-[6] and [8, 9, 15, 16, 21, 22, 23, 24, 25]. In this paper, we establish the Bullen type inequalities for conformable fractional integral and we will investigate some integral inequalities connected with Bullentype inequalities for conformable fractional integrals. The results presented here would provide generalizations of those given in earlier works. In this study, some new identity and Bullen type integral inequalities for differentiable functions are established and are applied to produce some inequalities of special means.

\section{Bullen Type Inequalities for Conformable Fractional Integral}

With the help of the following lemma, we will give some integral inequalities connected with Bullen-type inequalities for conformable fractional integral. 
Lemma 2 Let $\alpha \in(0,1]$ and $\Lambda: I \subset \mathbb{R}^{+} \rightarrow \mathbb{R}$ be an $\alpha$-fractional differentiable function on $\left(u_{1}, u_{2}\right)$ with $0 \leq u_{1}<u_{2}$. If $D_{\alpha}(\Lambda)$ be an $\alpha$-fractional integrable function on $\left[u_{1}^{\alpha}, u_{2}^{\alpha}\right]$, then the following identity for conformable fractional integral holds:

$$
\begin{aligned}
& \frac{1}{4} \int_{0}^{1}\left(1-2 t^{\alpha}\right)\left[D_{\alpha}(\Lambda)\left(\frac{1+t^{\alpha}}{2} u_{1}^{\alpha}+\frac{1-t^{\alpha}}{2} u_{2}^{\alpha}\right)\right. \\
& \left.+D_{\alpha}(\Lambda)\left(\frac{1+t^{\alpha}}{2} u_{2}^{\alpha}+\frac{1-t^{\alpha}}{2} u_{1}^{\alpha}\right)\right] d_{\alpha} t \\
= & \frac{\alpha}{u_{2}^{\alpha}-u_{1}^{\alpha}} \int_{u_{1}}^{u_{2}} \Lambda\left(x^{\alpha}\right) d_{\alpha} x-\frac{1}{2}\left[\frac{\Lambda\left(u_{1}^{\alpha}\right)+\Lambda\left(u_{2}^{\alpha}\right)}{2}+\Lambda\left(\frac{u_{1}^{\alpha}+u_{2}^{\alpha}}{2}\right)\right] .
\end{aligned}
$$

Proof. Integrating by parts

$$
\begin{aligned}
& \int_{0}^{1}\left(1-2 t^{\alpha}\right) D_{\alpha}(\Lambda)\left(\frac{1+t^{\alpha}}{2} u_{1}^{\alpha}+\frac{1-t^{\alpha}}{2} u_{2}^{\alpha}\right) d_{\alpha} t \\
& +\int_{0}^{1}\left(1-2 t^{\alpha}\right) D_{\alpha}(\Lambda)\left(\frac{1+t^{\alpha}}{2} u_{2}^{\alpha}+\frac{1-t^{\alpha}}{2} u_{1}^{\alpha}\right) d_{\alpha} t \\
= & \left.\left(1-2 t^{\alpha}\right) \Lambda\left(\frac{1+t^{\alpha}}{2} u_{1}^{\alpha}+\frac{1-t^{\alpha}}{2} u_{2}^{\alpha}\right)\right|_{0} ^{1} \\
& +2 \alpha \int_{0}^{1} \Lambda\left(\frac{1+t^{\alpha}}{2} u_{1}^{\alpha}+\frac{1-t^{\alpha}}{2} u_{2}^{\alpha}\right) d_{\alpha} t \\
& +\left.\left(1-2 t^{\alpha}\right) \Lambda\left(\frac{1+t^{\alpha}}{2} u_{2}^{\alpha}+\frac{1-t^{\alpha}}{2} u_{1}^{\alpha}\right)\right|_{0} ^{1} \\
& +2 \alpha \int_{0}^{1} \Lambda\left(\frac{1+t^{\alpha}}{2} u_{2}^{\alpha}+\frac{1-t^{\alpha}}{2} u_{1}^{\alpha}\right) d_{\alpha} t \\
= & -\Lambda\left(u_{1}^{\alpha}\right)-\Lambda\left(\frac{u_{1}^{\alpha}+u_{2}^{\alpha}}{2}\right)+\frac{4 \alpha}{u_{2}^{\alpha}-u_{1}^{\alpha}} \int_{u_{1}}^{\frac{u_{1}^{\alpha}+u_{2}^{\alpha}}{2}} \Lambda\left(x^{\alpha}\right) d_{\alpha} x \\
& -\Lambda\left(u_{2}^{\alpha}\right)-\Lambda\left(\frac{u_{1}^{\alpha}+u_{2}^{\alpha}}{2}\right)+\frac{4 \alpha}{u_{2}^{\alpha}-u_{1}^{\alpha}} \int_{\frac{u_{1}^{\alpha}+u_{2}^{\alpha}}{2}}^{u_{2}} \Lambda\left(x^{\alpha}\right) d_{\alpha} x \\
= & -\left[\Lambda\left(u_{1}^{\alpha}\right)+\Lambda\left(u_{2}^{\alpha}\right)+2 f\left(\frac{u_{1}^{\alpha}+u_{2}^{\alpha}}{2}\right)\right]^{u_{2}} \Lambda\left(x^{\alpha}\right) d_{\alpha} x . \\
& +\frac{4 \alpha}{u_{2}^{\alpha}-u_{1}^{\alpha}} \int_{u_{1}}
\end{aligned}
$$

Thus, proof is completed.

Theorem 9 Let $\alpha \in(0,1]$ and $\Lambda: I \subset R^{+} \rightarrow R$ be an $\alpha$-fractional differentiable function on $I^{\circ}$ and $D_{\alpha}(\Lambda)$ be an $\alpha$-fractional integrable function on $I$ with $0 \leq u_{1}<$ $u_{2}$. If $\left|\Lambda^{\prime}\right|$ be a convex function on $I$, then the following inequality for conformable fractional integral holds: 


$$
\begin{aligned}
& \left|\frac{\alpha}{u_{2}^{\alpha}-u_{1}^{\alpha}} \int_{u_{1}}^{u_{2}} \Lambda\left(x^{\alpha}\right) d_{\alpha} x-\frac{1}{2}\left[\frac{\Lambda\left(u_{1}^{\alpha}\right)+\Lambda\left(u_{2}^{\alpha}\right)}{2}+\Lambda\left(\frac{u_{1}^{\alpha}+u_{2}^{\alpha}}{2}\right)\right]\right| \\
\leq & \frac{5\left(u_{2}^{\alpha}-u_{1}^{\alpha}\right)}{128}\left(\left(u_{1}^{\alpha}\right)^{\alpha-1}\left|D_{\alpha}(\Lambda)\left(u_{1}^{\alpha}\right)\right|+\left(u_{2}^{\alpha}\right)^{\alpha-1}\left|D_{\alpha}(\Lambda)\left(u_{2}^{\alpha}\right)\right|\right) .
\end{aligned}
$$

Proof. Since $\left|\Lambda^{\prime}\right|$ is a convex function on $I$, by the using the properties $D_{\alpha}(\Lambda \circ g)(t)=$ $\Lambda^{\prime}(g(t)) D_{\alpha}(g(t))$ and $D_{\alpha}(\Lambda(t))=t^{1-\alpha} \Lambda^{\prime}(t)$ and by Lemma 2 and using the well known absolute value inequality, and we have

$$
\begin{aligned}
& \mid \frac{\alpha}{u_{2}^{\alpha}-u_{1}^{\alpha}} \int_{u_{1}}^{u_{2}} \Lambda\left(x^{\alpha}\right) d_{\alpha} x \\
& -\frac{1}{2}\left[\frac{\Lambda\left(u_{1}^{\alpha}\right)+\Lambda\left(u_{2}^{\alpha}\right)}{2}+\Lambda\left(\frac{u_{1}^{\alpha}+u_{2}^{\alpha}}{2}\right)\right] \mid \\
\leq & \frac{1}{4} \int_{0}^{1}\left|1-2 t^{\alpha}\right|\left|D_{\alpha}(\Lambda)\left(\frac{1+t^{\alpha}}{2} u_{1}^{\alpha}+\frac{1-t^{\alpha}}{2} u_{2}^{\alpha}\right)\right| d_{\alpha} t \\
& +\frac{1}{4} \int_{0}^{1}\left|1-2 t^{\alpha}\right|\left|D_{\alpha}(\Lambda)\left(\frac{1+t^{\alpha}}{2} u_{2}^{\alpha}+\frac{1-t^{\alpha}}{2} u_{1}^{\alpha}\right)\right| d_{\alpha} t \\
\leq & \frac{\alpha\left(u_{2}^{\alpha}-u_{1}^{\alpha}\right)}{8}\left(\left(u_{1}^{\alpha}\right)^{\alpha-1}\left|D_{\alpha}(\Lambda)\left(u_{1}^{\alpha}\right)\right| \int_{0}^{1}\left|1-2 t^{\alpha}\right| \frac{1+t^{\alpha}}{2} d_{\alpha} t\right) \\
& +\frac{\alpha\left(u_{2}^{\alpha}-u_{1}^{\alpha}\right)}{8}\left(\left(u_{2}^{\alpha}\right)^{\alpha-1}\left|D_{\alpha}(\Lambda)\left(u_{2}^{\alpha}\right)\right| \int_{0}^{1}\left|1-2 t^{\alpha}\right| \frac{1-t^{\alpha}}{2} d_{\alpha} t\right) \\
& +\frac{\alpha\left(u_{2}^{\alpha}-u_{1}^{\alpha}\right)}{8}\left(\left(u_{2}^{\alpha}\right)^{\alpha-1}\left|D_{\alpha}(\Lambda)\left(u_{2}^{\alpha}\right)\right| \int_{0}^{1}\left|1-2 t^{\alpha}\right| \frac{1+t^{\alpha}}{2} d_{\alpha} t\right) \\
& +\frac{\alpha\left(u_{2}^{\alpha}-u_{1}^{\alpha}\right)}{8}\left(\left(u_{1}^{\alpha}\right)^{\alpha-1}\left|D_{\alpha}(\Lambda)\left(u_{1}^{\alpha}\right)\right| \int_{0}^{1}\left|1-2 t^{\alpha}\right| \frac{1-t^{\alpha}}{2} d_{\alpha} t\right) \\
= & \frac{5\left(u_{2}^{\alpha}-u_{1}^{\alpha}\right)}{128}\left(\left(u_{1}^{\alpha}\right)^{\alpha-1}\left|D_{\alpha}(\Lambda)\left(u_{1}^{\alpha}\right)\right|+\left(u_{2}^{\alpha}\right)^{\alpha-1}\left|D_{\alpha}(\Lambda)\left(u_{2}^{\alpha}\right)\right|\right) .
\end{aligned}
$$

where

$$
\begin{aligned}
& \int_{0}^{1}\left|1-2 t^{\alpha}\right| \frac{1+t^{\alpha}}{2} d_{\alpha} t \\
= & \int_{0}^{1}\left|1-2 t^{\alpha}\right| \frac{1+t^{\alpha}}{2} t^{\alpha-1} d t \\
= & \int_{0}^{\frac{1}{2^{1 / \alpha}}}\left(1-2 t^{\alpha}\right) \frac{1+t^{\alpha}}{2} t^{\alpha-1} d t+\int_{\frac{1}{2^{1 / \alpha}}}^{1}\left(2 t^{\alpha}-1\right) \frac{1+t^{\alpha}}{2} t^{\alpha-1} d t \\
= & \frac{3}{16 \alpha}
\end{aligned}
$$


and

$$
\begin{aligned}
& \int_{0}^{1}\left|1-2 t^{\alpha}\right| \frac{1-t^{\alpha}}{2} d_{\alpha} t \\
= & \int_{0}^{1}\left|1-2 t^{\alpha}\right| \frac{1-t^{\alpha}}{2} t^{\alpha-1} d t \\
= & \int_{0}^{\frac{1}{2^{1 / \alpha}}}\left(1-2 t^{\alpha}\right) \frac{1-t^{\alpha}}{2} t^{\alpha-1} d t+\int_{\frac{1}{2^{1 / \alpha}}}^{1}\left(2 t^{\alpha}-1\right) \frac{1-t^{\alpha}}{2} t^{\alpha-1} d t \\
= & \frac{1}{8 \alpha} .
\end{aligned}
$$

Remark 2 If we choose $\alpha=1$ in Theorem 9, then we obtain following inequality:

$$
\begin{aligned}
& \left|\frac{1}{u_{2}-u_{1}} \int_{u_{1}}^{u_{2}} \Lambda(x) d x-\frac{1}{2}\left[\frac{\Lambda\left(u_{1}\right)+\Lambda\left(u_{2}\right)}{2}+\Lambda\left(\frac{u_{1}+u_{2}}{2}\right)\right]\right| \\
\leq & \frac{5\left(u_{2}-u_{1}\right)}{128}\left(\left|\Lambda^{\prime}\left(u_{1}\right)\right|+\left|\Lambda^{\prime}\left(u_{2}\right)\right|\right) .
\end{aligned}
$$

Theorem 10 Let $\alpha \in(0,1]$ and $\Lambda: I \subset R^{+} \rightarrow R$ be an $\alpha$-fractional differentiable function on $I^{\circ}$ and $D_{\alpha}(\Lambda)$ be an $\alpha$-fractional integrable function on $I$ with $0 \leq u_{1}<$ $u_{2}$ and $p, q>1,1 / p+1 / q=1$. If $\left|\Lambda^{\prime}\right|^{q}$ be a convex function on $I$, then the following inequality for conformable fractional integral holds:

$$
\begin{aligned}
& \left|\frac{\alpha}{u_{2}^{\alpha}-u_{1}^{\alpha}} \int_{u_{1}}^{u_{2}} \Lambda\left(x^{\alpha}\right) d_{\alpha} x-\frac{1}{2}\left[\frac{\Lambda\left(u_{1}^{\alpha}\right)+\Lambda\left(u_{2}^{\alpha}\right)}{2}+\Lambda\left(\frac{u_{1}^{\alpha}+u_{2}^{\alpha}}{2}\right)\right]\right| \\
\leq & \frac{\alpha\left(u_{2}^{\alpha}-u_{1}^{\alpha}\right)}{8}\left(\frac{1}{\alpha(1+p)}\right)^{\frac{1}{p}}\left(\frac{1}{4 \alpha}\right)^{\frac{1}{q}} \\
& \times\left[\left(3\left(u_{1}^{q \alpha}\right)^{\alpha-1}\left|D_{\alpha}(\Lambda)\left(u_{1}^{\alpha}\right)\right|^{q}+\left(u_{2}^{q \alpha}\right)^{\alpha-1}\left|D_{\alpha}(\Lambda)\left(u_{2}^{\alpha}\right)\right|^{q}\right)^{\frac{1}{q}}\right. \\
& \left.+\left(\left(u_{1}^{q \alpha}\right)^{\alpha-1}\left|D_{\alpha}(\Lambda)\left(u_{1}^{\alpha}\right)\right|^{q}+3\left(u_{2}^{q \alpha}\right)^{\alpha-1}\left|D_{\alpha}(\Lambda)\left(u_{2}^{\alpha}\right)\right|^{q}\right)^{\frac{1}{q}}\right] .
\end{aligned}
$$

Proof. Since $\left|\Lambda^{\prime}\right|^{q}$ is a convex function on $I$, by the using the properties $D_{\alpha}(\Lambda \circ g)(t)=$ $\Lambda^{\prime}(g(t)) D_{\alpha}(g(t)), D_{\alpha}(\Lambda(t))=t^{1-\alpha} \Lambda^{\prime}(t)$, by Lemma 2 and using the well known Hölder inequality, we have

$$
\begin{aligned}
& \left|\frac{\alpha}{u_{2}^{\alpha}-u_{1}^{\alpha}} \int_{u_{1}}^{u_{2}} \Lambda\left(x^{\alpha}\right) d_{\alpha} x-\frac{1}{2}\left[\frac{\Lambda\left(u_{1}^{\alpha}\right)+\Lambda\left(u_{2}^{\alpha}\right)}{2}+\Lambda\left(\frac{u_{1}^{\alpha}+u_{2}^{\alpha}}{2}\right)\right]\right| \\
\leq & \frac{1}{4}\left(\int_{0}^{1}\left(1-2 t^{\alpha}\right)^{p} d_{\alpha} t\right)^{\frac{1}{p}}\left(\int_{0}^{1}\left(D_{\alpha}(\Lambda)\left(\frac{1+t^{\alpha}}{2} u_{1}^{\alpha}+\frac{1-t^{\alpha}}{2} u_{2}^{\alpha}\right)\right)^{q} d_{\alpha} t\right)^{\frac{1}{q}} \\
& +\frac{1}{4}\left(\int_{0}^{1}\left(1-2 t^{\alpha}\right)^{p} d_{\alpha} t\right)^{\frac{1}{p}}\left(\int_{0}^{1}\left(D_{\alpha}(\Lambda)\left(\frac{1+t^{\alpha}}{2} u_{2}^{\alpha}+\frac{1-t^{\alpha}}{2} b a^{\alpha}\right)\right)^{q} d_{\alpha} t\right)^{\frac{1}{q}}
\end{aligned}
$$




$$
\begin{aligned}
\leq & \frac{\alpha\left(u_{2}^{\alpha}-u_{1}^{\alpha}\right)}{8}\left(\frac{1}{\alpha(1+p)}\right)^{\frac{1}{p}} \\
& \times\left(\frac{3}{4 \alpha}\left(u_{1}^{q \alpha}\right)^{\alpha-1}\left|D_{\alpha}(\Lambda)\left(u_{1}^{\alpha}\right)\right|^{q}+\frac{1}{4 \alpha}\left(u_{2}^{q \alpha}\right)^{\alpha-1}\left|D_{\alpha}(\Lambda)\left(u_{2}^{\alpha}\right)\right|^{q}\right)^{\frac{1}{q}} \\
& +\frac{\alpha\left(u_{2}^{\alpha}-u_{1}^{\alpha}\right)}{8}\left(\frac{1}{\alpha(1+p)}\right)^{\frac{1}{p}} \\
& \times\left(\frac{3}{4 \alpha}\left(u_{2}^{q \alpha}\right)^{\alpha-1}\left|D_{\alpha}(\Lambda)\left(u_{2}^{\alpha}\right)\right|^{q}+\frac{1}{4 \alpha}\left(u_{1}^{q \alpha}\right)^{\alpha-1}\left|D_{\alpha}(\Lambda)\left(u_{1}^{\alpha}\right)\right|^{q}\right)^{\frac{1}{q}} \\
= & \frac{\alpha\left(u_{2}^{\alpha}-u_{1}^{\alpha}\right)}{8}\left(\frac{1}{\alpha(1+p)}\right)^{\frac{1}{p}}\left(\frac{1}{4 \alpha}\right)^{\frac{1}{q}} \\
& \times\left[\left(3\left(u_{1}^{q \alpha}\right)^{\alpha-1}\left|D_{\alpha}(\Lambda)\left(u_{1}^{\alpha}\right)\right|^{q}+\left(u_{2}^{q \alpha}\right)^{\alpha-1}\left|D_{\alpha}(\Lambda)\left(u_{2}^{\alpha}\right)\right|^{q}\right)^{\frac{1}{q}}\right. \\
& \left.+\left(\left(u_{1}^{q \alpha}\right)^{\alpha-1}\left|D_{\alpha}(\Lambda)\left(u_{1}^{\alpha}\right)\right|^{q}+3\left(u_{2}^{q \alpha}\right)^{\alpha-1}\left|D_{\alpha}(\Lambda)\left(u_{2}^{\alpha}\right)\right|^{q}\right)^{\frac{1}{q}}\right] .
\end{aligned}
$$

where

$$
\begin{aligned}
\int_{0}^{1}\left(1-2 t^{\alpha}\right)^{p} d_{\alpha} t & =\int_{0}^{1}\left(1-2 t^{\alpha}\right)^{p} t^{\alpha-1} d t \\
& =\int_{0}^{\frac{1}{2^{1 / \alpha}}}\left(1-2 t^{\alpha}\right)^{p} t^{\alpha-1} d t+\int_{\frac{1}{2^{1 / \alpha}}}^{1}\left(2 t^{\alpha}-1\right)^{p} t^{\alpha-1} d t \\
& =\frac{1}{\alpha(1+p)}
\end{aligned}
$$

and

$$
\begin{aligned}
& \int_{0}^{1} \frac{1+t^{\alpha}}{2} d_{\alpha} t=\int_{0}^{1} \frac{1+t^{\alpha}}{2} t^{\alpha-1} d t=\frac{3}{4 \alpha} \\
& \int_{0}^{1} \frac{1-t^{\alpha}}{2} d_{\alpha} t=\int_{0}^{1} \frac{1-t^{\alpha}}{2} t^{\alpha-1} d t=\frac{1}{4 \alpha} .
\end{aligned}
$$

Remark 3 If we choose $\alpha=1$ in Theorem 10, then we obtain following inequality:

$$
\begin{aligned}
& \left|\frac{1}{u_{2}-u_{1}} \int_{u_{1}}^{u_{2}} \Lambda(x) d x-\frac{1}{2}\left[\frac{\Lambda\left(u_{1}\right)+\Lambda\left(u_{2}\right)}{2}+\Lambda\left(\frac{u_{1}+u_{2}}{2}\right)\right]\right| \\
\leq & \frac{\left(u_{2}-u_{1}\right)}{8}\left(\frac{1}{(1+p)}\right)^{\frac{1}{p}}\left(\frac{1}{4}\right)^{\frac{1}{q}} \\
& \times\left[\left(3\left(\Lambda^{\prime}\left(u_{1}\right)\right)^{q}+\left(\Lambda^{\prime}\left(u_{2}\right)\right)^{q}\right)^{\frac{1}{q}}+\left(\left(\Lambda^{\prime}\left(u_{1}\right)\right)^{q}+3\left(\Lambda^{\prime}\left(u_{2}\right)\right)^{q}\right)^{\frac{1}{q}}\right] .
\end{aligned}
$$

Remark 4 If we choose $\alpha=1$ in Theorem 10, then we obtain Corollary 1. 
Theorem 11 Let $\alpha \in(0,1]$ and $\Lambda: I \subset R^{+} \rightarrow R$ be an $\alpha$-fractional differentiable function on $I^{\circ}$ and $D_{\alpha}(\Lambda)$ be an $\alpha$-fractional integrable function on $I$ with $0 \leq u_{1}<$ $u_{2}$ and $q \geq 1$. If $\left|\Lambda^{\prime}\right|^{q}$ be a convex function on $I$, then the following inequality for conformable fractional integral holds:

$$
\begin{aligned}
& \left|\frac{\alpha}{u_{2}^{\alpha}-u_{1}^{\alpha}} \int_{u_{1}}^{u_{2}} \Lambda\left(x^{\alpha}\right) d_{\alpha} x-\frac{1}{2}\left[\frac{\Lambda\left(u_{1}^{\alpha}\right)+\Lambda\left(u_{2}^{\alpha}\right)}{2}+\Lambda\left(\frac{u_{1}^{\alpha}+u_{2}^{\alpha}}{2}\right)\right]\right| \\
\leq & \frac{\left(u_{2}^{\alpha}-u_{1}^{\alpha}\right)}{16}\left(\frac{1}{4}\right)^{\frac{1}{q}} \\
& \times\left[\left(3\left(u_{1}^{q \alpha}\right)^{\alpha-1}\left|D_{\alpha}(\Lambda)\left(u_{1}^{\alpha}\right)\right|^{q}+\left(u_{2}^{q \alpha}\right)^{\alpha-1}\left|D_{\alpha}(\Lambda)\left(u_{2}^{\alpha}\right)\right|^{q}\right)^{\frac{1}{q}}\right. \\
& \left.+\left(3\left(u_{2}^{q \alpha}\right)^{\alpha-1}\left|D_{\alpha}(\Lambda)\left(u_{2}^{\alpha}\right)\right|^{q}+\left(u_{1}^{q \alpha}\right)^{\alpha-1}\left|D_{\alpha}(\Lambda)\left(u_{1}^{\alpha}\right)\right|^{q}\right)^{\frac{1}{q}}\right] .
\end{aligned}
$$

Proof. Since $\left|\Lambda^{\prime}\right|^{q}$ is a convex function on $I$, by the using the properties $D_{\alpha}(\Lambda \circ g)(t)=$ $\Lambda^{\prime}(g(t)) D_{\alpha}(g(t))$ and $D_{\alpha}(\Lambda(t))=t^{1-\alpha} \Lambda^{\prime}(t)$ and assume that $q \geq 1$, by Lemma 2 and using the well known power-mean inequality, we have

$$
\begin{aligned}
& \left|\frac{\alpha}{u_{2}^{\alpha}-u_{1}^{\alpha}} \int_{u_{1}}^{u_{2}} \Lambda\left(x^{\alpha}\right) d_{\alpha} x-\frac{1}{2}\left[\frac{\Lambda\left(u_{1}^{\alpha}\right)+\Lambda\left(u_{2}^{\alpha}\right)}{2}+\Lambda\left(\frac{u_{1}^{\alpha}+u_{2}^{\alpha}}{2}\right)\right]\right| \\
\leq & \frac{\alpha\left(u_{2}^{\alpha}-u_{1}^{\alpha}\right)}{8}\left(\int_{0}^{1}\left|1-2 t^{\alpha}\right| d_{\alpha} t\right)^{1-\frac{1}{q}}\left(\left(u_{1}^{q \alpha}\right)^{\alpha-1}\left|D_{\alpha}(\Lambda)\left(u_{1}^{\alpha}\right)\right|^{q} \int_{0}^{1}\left|1-2 t^{\alpha}\right| \frac{1+t^{\alpha}}{2} d_{\alpha} t\right. \\
& \left.+\left(u_{2}^{q \alpha}\right)^{\alpha-1}\left|D_{\alpha}(\Lambda)\left(u_{2}^{\alpha}\right)\right|^{q} \int_{0}^{1}\left|1-2 t^{\alpha}\right| \frac{1-t^{\alpha}}{2} d_{\alpha} t\right)^{\frac{1}{q}} \\
& +\frac{\alpha\left(u_{2}^{\alpha}-u_{1}^{\alpha}\right)}{8}\left(\int_{0}^{1}\left|1-2 t^{\alpha}\right| d_{\alpha} t\right)^{1-\frac{1}{q}}\left(\left(u_{2}^{q \alpha}\right)^{\alpha-1}\left|D_{\alpha}(\Lambda)\left(u_{2}^{\alpha}\right)\right|^{q} \int_{0}^{1}\left|1-2 t^{\alpha}\right| \frac{1+t^{\alpha}}{2} d_{\alpha} t\right. \\
& \left.+\left(u_{1}^{q \alpha}\right)^{\alpha-1}\left|D_{\alpha}(\Lambda)\left(u_{1}^{\alpha}\right)\right|^{q} \int_{0}^{1}\left|1-2 t^{\alpha}\right| \frac{1-t^{\alpha}}{2} d_{\alpha} t\right)^{\frac{1}{q}} \\
= & \frac{\alpha\left(u_{2}^{\alpha}-u_{1}^{\alpha}\right)}{8}\left(\frac{1}{2 \alpha}\right)^{1-\frac{1}{q}}\left(\frac{2}{16 \alpha}\right)^{\frac{1}{q}} \\
& \cdot\left(3\left(u_{1}^{q \alpha}\right)^{\alpha-1}\left|D_{\alpha}(\Lambda)\left(u_{1}^{\alpha}\right)\right|^{q}+\left(u_{2}^{q \alpha}\right)^{\alpha-1}\left|D_{\alpha}(\Lambda)\left(u_{2}^{\alpha}\right)\right|^{q}\right)^{\frac{1}{q}} \\
& +\frac{\alpha\left(u_{2}^{\alpha}-u_{1}^{\alpha}\right)}{8}\left(\frac{1}{2 \alpha}\right)^{1-\frac{1}{q}}\left(\frac{2}{16 \alpha}\right)^{\frac{1}{q}} \\
& \cdot\left(3\left(u_{2}^{q \alpha}\right)^{\alpha-1}\left|D_{\alpha}(\Lambda)\left(u_{2}^{\alpha}\right)\right|^{q}+\left(u_{1}^{q \alpha}\right)^{\alpha-1}\left|D_{\alpha}(\Lambda)\left(u_{1}^{\alpha}\right)\right|^{q}\right)^{\frac{1}{q}}
\end{aligned}
$$




$$
\begin{aligned}
= & \frac{\left(u_{2}^{\alpha}-u_{1}^{\alpha}\right)}{16}\left(\frac{1}{4}\right)^{\frac{1}{q}} \times \\
& {\left[\left(3\left(u_{1}^{q \alpha}\right)^{\alpha-1}\left|D_{\alpha}(\Lambda)\left(u_{1}^{\alpha}\right)\right|^{q}+\left(u_{2}^{q \alpha}\right)^{\alpha-1}\left|D_{\alpha}(\Lambda)\left(u_{2}^{\alpha}\right)\right|^{q}\right)^{\frac{1}{q}}\right.} \\
& \left.+\left(3\left(u_{2}^{q \alpha}\right)^{\alpha-1}\left|D_{\alpha}(\Lambda)\left(u_{2}^{\alpha}\right)\right|^{q}+\left(u_{1}^{q \alpha}\right)^{\alpha-1}\left|D_{\alpha}(\Lambda)\left(u_{1}^{\alpha}\right)\right|^{q}\right)^{\frac{1}{q}}\right] .
\end{aligned}
$$

where

$$
\int_{0}^{1}\left|1-2 t^{\alpha}\right| d_{\alpha} t=\int_{0}^{\frac{1}{2^{1 / \alpha}}}\left(1-2 t^{\alpha}\right) t^{\alpha-1} d t+\int_{\frac{1}{2^{1 / \alpha}}}^{1}\left(2 t^{\alpha}-1\right) t^{\alpha-1} d t=\frac{1}{2 \alpha}
$$

and

$$
\begin{aligned}
& \int_{0}^{1}\left|1-2 t^{\alpha}\right| \frac{1+t^{\alpha}}{2} d_{\alpha} t \\
= & \int_{0}^{\frac{1}{2^{1 / \alpha}}}\left(1-2 t^{\alpha}\right) \frac{1+t^{\alpha}}{2} t^{\alpha-1} d t+\int_{\frac{1}{2^{1 / \alpha}}}^{1}\left(2 t^{\alpha}-1\right) \frac{1+t^{\alpha}}{2} t^{\alpha-1} d t \\
= & \frac{3}{8 \alpha}, \\
= & \int_{0}^{\frac{1}{2^{1 / \alpha}}}\left(1-2 t^{\alpha}\right) \frac{1-t^{\alpha}}{2} t^{\alpha-1} d t+\int_{\frac{1}{2^{1 / \alpha}}}^{1}\left(2 t^{\alpha}-1\right) \frac{1-t^{\alpha}}{2} t^{\alpha-1} d t \\
= & \frac{1}{8 \alpha} .
\end{aligned}
$$

Remark 5 If we choose $\alpha=1$ in Theorem 11, then we obtain following inequality:

$$
\begin{aligned}
& \left|\frac{1}{u_{2}-u_{1}} \int_{u_{1}}^{u_{2}} \Lambda(x) d x-\frac{1}{2}\left[\frac{\Lambda\left(u_{1}\right)+\Lambda\left(u_{2}\right)}{2}+\Lambda\left(\frac{u_{1}+u_{2}}{2}\right)\right]\right| \\
\leq & \frac{\left(u_{2}-u_{1}\right)}{16}\left(\frac{1}{4}\right)^{\frac{1}{q}} \times \\
& {\left[\left(3\left(\Lambda^{\prime}\left(u_{1}\right)\right)^{q}+\left(\Lambda^{\prime}\left(u_{2}\right)\right)^{q}\right)^{\frac{1}{q}}+\left(\left(\Lambda^{\prime}\left(u_{1}\right)\right)^{q}+3\left(\Lambda^{\prime}\left(u_{2}\right)\right)^{q}\right)^{\frac{1}{q}}\right] . }
\end{aligned}
$$

Remark 6 If we choose $\alpha=1$ in Theorem 11, then we obtain Corollary 2. 


\section{Applications}

Let

$$
\begin{aligned}
A\left(u_{1}, u_{2}\right) & =\frac{u_{1}+u_{2}}{2} \\
G\left(u_{1}, u_{2}\right) & =\sqrt{u_{1} u_{2}}, \\
L_{p}\left(u_{1}, u_{2}\right) & =\left(\frac{u_{2}^{p+1}-u_{1}^{p+1}}{(p+1)\left(u_{2}-u_{1}\right)}\right)^{1 / p}, u_{1} \neq u_{2}, p \in \mathbb{R}, p \neq-1,0
\end{aligned}
$$

be the arithmetic mean, geometric mean, generalized logarithmic mean for $u_{1}, u_{2}>0$ respectively.

Proposition 1 Let $s \in(0,1], u_{1}, u_{2}>0$, then

$$
\begin{aligned}
& \quad\left|\frac{\alpha\left(u_{2}^{n a+\alpha}-u_{1}^{n a+\alpha}\right)}{\left(n u_{1}+\alpha\right)\left(u_{2}^{\alpha}-u_{1}^{\alpha}\right)}-\frac{1}{2}\left[A\left(u_{1}^{n \alpha}, u_{2}^{n \alpha}\right)+A^{n}\left(u_{1}^{\alpha}, u_{2}^{\alpha}\right)\right]\right| \\
& \leq \frac{5\left(u_{2}^{\alpha}-u_{1}^{\alpha}\right)}{128}\left(n\left(u_{1}^{\alpha}\right)^{n-1}+n\left(u_{2}^{\alpha}\right)^{n-1}\right) .
\end{aligned}
$$

Proof. The claim follows from Theorem 9 applied to convex function $\Lambda(\varkappa)=\varkappa^{n}$ where $n \in \mathbb{N}$.

Example 1 If we take $\alpha=1, n=2$ in Proposition 1, then we can obtain following inequality:

$$
\left|\frac{2 A\left(u_{1}^{2}, u_{2}^{2}\right)+G^{2}\left(u_{1}, u_{2}\right)}{\left(2 u_{1}+1\right)}-A\left[A\left(u_{1}^{2}, u_{2}^{2}\right), A^{2}\left(u_{1}, u_{2}\right)\right]\right| \leq \frac{5\left(u_{2}^{2}-u_{1}^{2}\right)}{64} .
$$

Proposition 2 Let $\alpha \in(0,1], u_{1}, u_{2}>0, p, q>1$, then

$$
\begin{aligned}
& \left|\frac{\alpha\left(u_{2}^{n a+\alpha}-u_{1}^{n a+\alpha}\right)}{\left(n u_{1}+\alpha\right)\left(u_{2}^{\alpha}-u_{1}^{\alpha}\right)}-\frac{1}{2}\left[A\left(u_{1}^{n \alpha}, u_{2}^{n \alpha}\right)+A^{n}\left(u_{1}^{\alpha}, u_{2}^{\alpha}\right)\right]\right| \\
\leq & \frac{\alpha\left(u_{2}^{\alpha}-u_{1}^{\alpha}\right)}{8}\left(\frac{1}{\alpha(1+p)}\right)^{\frac{1}{p}}\left(\frac{1}{4 \alpha}\right)^{\frac{1}{q}} \times \\
& {\left[\left(3\left(n u_{1}^{\alpha(n-1)}\right)^{q}+\left(n u_{2}^{\alpha(n-1)}\right)^{q}\right)^{\frac{1}{q}}\right.} \\
& \left.+\left(\left(n u_{1}^{\alpha(n-1)}\right)^{q}+3\left(n u_{2}^{\alpha(n-1)}\right)^{q}\right)^{\frac{1}{q}}\right] .
\end{aligned}
$$

Proof. The claim follows from Theorem 10 applied to convex $\Lambda(\varkappa)=\varkappa^{n}$ where $n \in \mathbb{N}$. 
Example 2 If we take $\alpha=1, p=q=n=2$ in Proposition 2, then we can obtain following inequality:

$$
\begin{aligned}
& \left|\frac{2 A\left(u_{1}^{2}, u_{2}^{2}\right)+G^{2}\left(u_{1}, u_{2}\right)}{\left(2 u_{1}+1\right)}-A\left[A\left(u_{1}^{2}, u_{2}^{2}\right), A^{2}\left(u_{1}, u_{2}\right)\right]\right| \\
\leq & \frac{\left(u_{2}-u_{1}\right)}{4}\left(\frac{1}{12}\right)^{\frac{1}{2}} \times\left[\left(3 u_{1}^{2}+u_{2}^{2}\right)^{\frac{1}{2}}+\left(3 u_{2}^{2}+u_{1}^{2}\right)^{\frac{1}{2}}\right] .
\end{aligned}
$$

Proposition 3 Let $\alpha \in(0,1], u_{1}, u_{2}>0, q \geq 1$, then

$$
\begin{aligned}
& \left|\frac{\alpha\left(u_{2}^{n a+\alpha}-u_{1}^{n a+\alpha}\right)}{\left(n u_{1}+\alpha\right)\left(u_{2}^{\alpha}-u_{1}^{\alpha}\right)}-\frac{1}{2}\left[A\left(u_{1}^{n \alpha}, u_{2}^{n \alpha}\right)+A^{n}\left(u_{1}^{\alpha}, u_{2}^{\alpha}\right)\right]\right| \\
\leq & \frac{u_{2}^{\alpha}-u_{1}^{\alpha}}{16}\left(\frac{1}{4}\right)^{\frac{1}{q}} \times \\
& {\left[\left(3\left(n u_{1}^{\alpha(n-1)}\right)^{q}+\left(n u_{2}^{\alpha(n-1)}\right)^{q}\right)^{\frac{1}{q}}\right.} \\
& \left.+\left(\left(n u_{1}^{\alpha(n-1)}\right)^{q}+3\left(n u_{2}^{\alpha(n-1)}\right)^{q}\right)^{\frac{1}{q}}\right] .
\end{aligned}
$$

Proof. The claim follows from Theorem 11 applied to convex $\Lambda(\varkappa)=\varkappa^{n}$ where $n \in \mathbb{N}$.

Example 3 If we take $\alpha=1, q=2, n=2$ in Proposition 3, then we can obtain following inequality:

$$
\begin{aligned}
& \left|\frac{2 A\left(u_{1}^{2}, u_{2}^{2}\right)+G^{2}\left(u_{1}, u_{2}\right)}{\left(2 u_{1}+1\right)}-A\left[A\left(u_{1}^{2}, u_{2}^{2}\right), A^{2}\left(u_{1}, u_{2}\right)\right]\right| \\
\leq & \frac{u_{2}-u_{1}}{32} \times\left[\left(3 u_{1}^{2}+u_{2}^{2}\right)^{\frac{1}{2}}+\left(u_{1}^{2}+3 u_{2}^{2}\right)^{\frac{1}{2}}\right] .
\end{aligned}
$$

\section{References}

[1] M. Abu Hammad, R. Khalil, Conformable fractional heat differential equations, International Journal of Pure and Applied Mathematics, vol. 94, no. 2, 2014, 215-221, doi:10.12732/ijpam.v94i2.8.

[2] M. Abu Hammad, R. Khalil, Abel's formula and wronskian for conformable fractional differential equations, International Journal of Differential Equations and Applications, vol. 13, no. 3, 2014, 177-183, doi:10.12732/ijdea.v 13i3.1753.

[3] A. Akkurt, Me. Yıldırım, H. Yıldırım, On some integral inequalities for conformable fractional integrals, Asian Journal of Mathematics and Computer Research, vol. 15, 2017, 205-212. 
[4] A. Akkurt, Me. Yıldırım, H. Yıldırım, A new generalized fractional derivative and integral, Konuralp Journal of Mathematics, vol. 5, no. 2, 2017, 248-259.

[5] T. Abdeljawad, On conformable fractional calculus. Journal of Computational and Applied Mathematics, vol. 279, 2015, 57-66.

[6] D. R. Anderson, P. Pardalos, T. Rassias, Taylors formula and integral inequalities for conformable fractional derivatives. Contributions in Mathematics and Engineering. Springer, Cham, 2016, 25-43, https://doi.org/10.1007/978-3-319-31317$7-2$.

[7] M. Bessenyei, Z. Páles, Characterization of convexity via Hadamard's inequality, Math. Inequal. Appl., vol. 9, 2006, 53-62.

[8] H. Budak, F. Usta, Mz. Sarikaya, Me. Ozdemir, On generalization of midpoint type inequalities with generalized fractional integral operators, Revista de la Real Academia de Ciencias Exactas, Fsicas y Naturales, Serie A, Matemticas, 2018, https://doi.org/10.1007/s13398-018-0514-z.

[9] H. Budak, F. Usta, Mz. Sarikaya, Some Weighted Integral Inequalities for Generalized Conformable Fractional Calculus, Iranian Journal of Mathematical Sciences and Informatics, accepted in 2018, in press.

[10] M. Çakmak, Some Hadamard and Bullen-type inequalities via s-convexity and their applications, 2018, submitted.

[11] S. S. Dragomir, R. P. Agarwal, P. Cerone, On Simpson's inequality and applications, J. of Ineq. and Appl., vol. 5, 2000, 533-579.

[12] S. S. Dragomir, Cem. Pearce,Selected topics on Hermite-Hadamard inequalities and applications, RGMIA monographs, Victoria University, 2000, http://www.staff.vu.edu.au/RGMIA/monographs/hermite-hadamard.html.

[13] J. Hadamard, Étude sur les propriétés des fonctions entières en particulier d'une fonction considérée par Riemann, J. Math. Pures Appl., vol. 58, 1893, 171-215.

[14] H. Hudzik, L. Maligranda, Some remarks on s-convex functions, Aequationes Math., vol. 48, 1994, 100-111.

[15] O. S. Iyiola, E. R.Nwaeze, Some new results on the new conformable fractional calculus with application using D'Alambert approach, Progr. Fract. Differ. Appl., vol. 2, no. 2, 2016, 115-122.

[16] R. Khalil, M. Al Horani, A. Yousef, M. Sababheh, A new definition of fractional derivative, Journal of Computational Applied Mathematics, vol. 264, 2014, 6570 .

[17] D. S. Mitrinović, I. B. Lacković, Hermite and convexity, Aequationes Math., vol. $28,1985,229-232$. 
[18] D. S. Mitrinović, J. E. Pecaric, A. M. Fink, Classical and New Inequalities in Analysis, Kluwer Academic Publishers, 1993.

[19] C. P. Niculescu, L. Persson, Convex Functions and their Applications, A Contemporary Approach, in: CMS Books in Mathematics, 23, Springer-Verlag, New York, 2006.

[20] M. Z. Sarikaya, E. Set, M. E. Özdemir, On new inequalities of Simpson's type for convex functions, RGMIA Res. Rep. Coll., vol. 13, no. 2, 2010, Article 2.

[21] F. Usta, H. Budak, M. Z. Sarikaya, E. Set, On generalization of trapezoid type inequalities for s-convex functions with generalized fractional integral operators, Filomat, vol. 32, no. 6, 2018, 2153-2171.

[22] F. Usta, M. Z. Sarikaya On generalization conformable fractional integral inequalities, FILOMAT, vol. 32, no. 16, 2018, 5519-5526.

[23] F. Usta, M. Z. Sarikaya, Explicit bounds on certain integral inequalities via conformable fractional calculus, Cogent Mathematics, vol. 4, no. 1, 2017, 1-8, doi: 10.1080/23311835.2016.1277505.

[24] F. Usta, M. Z. Sarikaya, Some Improvements of Comformable Fractional Integral inequalities, International Journal of Analysis and Applications, vol. 14, no. 2, 2017, 162-166.

[25] F. Usta, H. Budak, T. Tunç, M. Z. Sarikaya, New Bounds for the Ostrowski Type Inequalities via Conformable Fractionals Calculus, Arabian Journal of Mathematics, vol. 7, 2018, 317-328, doi:10.1007/s40065-018-0201-0.

[26] S. Wu, L. Debnath, Inequalities for convex sequences and their applications, Comp\&Math Appl., vol. 54, 2007, 525-534.

[27] B. Xi, F. Qi, Some Integral Inequalities of Hermite-Hadamard Type for Convex Functions with Applications to Means, Journal of Function Spaces and Appl., vol. 2012, Article ID 980438, doi:10.1155/2012/980438.

\section{Musa Çakmak}

Hatay Mustafa Kemal University

Reyhanl Vocational School, Turkey

e-mail: enkucukcakmak@gmail.com 